\title{
Reliability Analysis of the main shaft of the Nuclear Power CNC Wheel Milling Machine Based on the Probability Finite Element Method
}

\author{
Zhiwei $\mathrm{Li}^{1,}$ a \\ ${ }^{1}$ School of Sichuan College of Architectural Technology, De Yang Sichuan 618000, China \\ a814976273@qq.com
}

Keywords: nuclear power CNC wheel groove milling machine spindle, probability finite element, Monte Carlo simulation, reliability analysis

Abstract. In view of the complex factors affecting the reliability of the main shaft of a nuclear power CNC wheel milling machine, a finite element analysis model is established by using the ANSYS parametric modeling technique and the finite element analysis model of the machine tool spindle, which will affect the reliability of the main shaft of the machine tool, and the Carlo Monte simulation method is used to calculate the reliability. Get in the confidence probability of 95\%, three random variables of design variables histogram, failure probability distribution function, cumulative distribution curve and sensitivity analysis. The method proposed a new way of thinking for the reliability analysis and design of machine tool spindle, which has a great application prospect.

\section{Introduction}

With the continuous development of China's manufacturing industry, the research and development of ultra large equipment and the national attention, the domestic production of nuclear power CNC wheel groove milling machine, for China's power generation equipment independent manufacturing technology to promote and support role, and the spindle is the key part of the nuclear power plant, the reliability of the high level of nuclear power, so that only the machine tool spindle has high reliability, can guarantee the processing quality of the machine.

Because of the complex metal material and material characteristics of the main shaft of the machine tool spindle, the spindle power and torque are not the same. Therefore, it must take into account that the spindle head diameter, the elastic modulus and the torque of three random variables must be considered in the analysis. Aiming at this characteristic, the reliability analysis of the main shaft of the nuclear power wheel groove milling machine is proposed based on the probability finite element method.

\section{The finite element model of the main shaft}

The main shaft of the nuclear power CNC wheel groove milling machine is under the action of large torque. The torque of the main shaft is $8000 \mathrm{~N} . \mathrm{m}$. Therefore, the reliability of the rotor is considered in the design of the main shaft. The principal axis of the material is a composite metal material, the elastic modulus 200GP, the density is $(6.24 \sim 6.15) * 103 \mathrm{~kg} / \mathrm{m} 3$, the Poisson's ratio is 0.57 , the other main shaft key parts of the geometric parameters are shown in tab1.

Table.1 Geometric parameters of key parts of the spindle

\begin{tabular}{ccccc}
\hline $\begin{array}{c}\text { Parameter } \\
\text { variable }\end{array}$ & $\begin{array}{c}\text { Dimension parameter } \\
\text { description }\end{array}$ & Mean $/ \mathrm{mm}$ & Deviation $/ \mathrm{mm}$ & Under deviation $/ \mathrm{mm}$ \\
\hline 1 & $\begin{array}{c}\text { Inner diameter of } \\
\text { bearing inner ring }\end{array}$ & 580 & 0 & -0.012 \\
\hline 2 & $\begin{array}{c}\text { Bearing inner ring } \\
\text { outer diameter }\end{array}$ & 880 & 0 & -0.015 \\
\hline 3 & Bearing width & 710 & 0 & -0.12 \\
\hline 4 & Shaft length & 1100 & +0.001 & -0.001 \\
\hline 5 & $\begin{array}{c}\text { The inner diameter of } \\
\text { the shaft }\end{array}$ & 320 & +0.001 & -0.001 \\
\hline
\end{tabular}




\begin{tabular}{ccccc}
\hline 6 & $\begin{array}{c}\text { The outer diameter of } \\
\text { the shaft }\end{array}$ & 500 & +0.001 & -0.001 \\
\hline 7 & $\begin{array}{c}\text { The outer diameter of } \\
\text { the shaft }\end{array}$ & 430 & +0.001 & -0.001 \\
\hline
\end{tabular}

According to the basic size of the spindle, ignoring the impact of the main axis of the main axis of the main characteristics, the use of ANSYS/APDL to establish the parameters of the spindle model, the main axis of the whole grid using MESH200 and SOLID45 eight node solid element by multiple sweeping, the spindle and bearing of the wheel groove milling machine simplified, its finite element model is shown in fig 1.

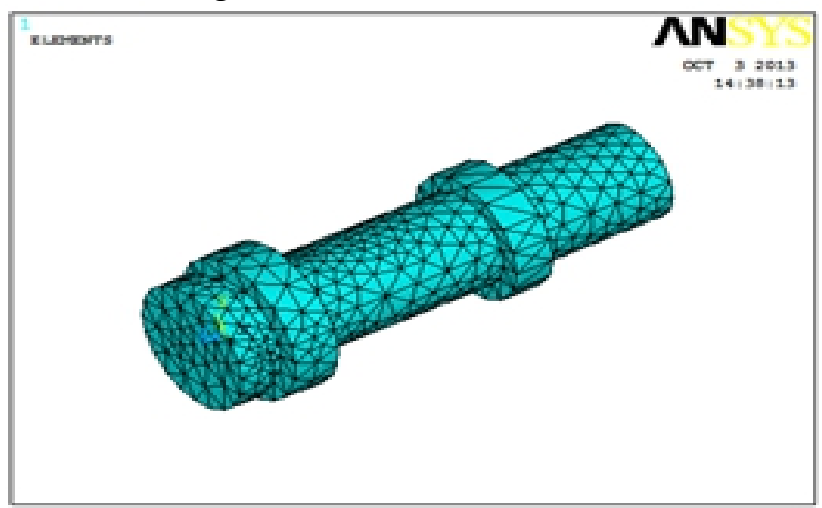

Fig.1 Finite element model of the spindle of the nuclear power wheel groove milling machine

\section{Monte Carlo simulation theory}

Monte Carlo simulation theory is the most common method in finite element analysis, which can clearly simulate the real behavior of the real problem. The basic idea of Monte Carlo simulation is as follows: the establishment of a random model corresponding to the research object, the formation of a random variable, it's a number of features (such as probability, expectation, etc.), and then in accordance with the random model of a large number of random experiments to obtain a large number of random variables, the use of statistical characteristics of the estimated value of the number of features. Based on this idea, the objective function is set up $Z=g\left(X_{1}, X_{2} \cdots, X_{n}\right)$, which $X_{\mathrm{i}}$ is a random variable with a certain distribution..

$X_{i}(i=1,2, \cdots, N)$ group was obtained by random sampling of $X_{i}^{j}(j=1,2, \cdots, n)$.The group $\mathrm{J}$ values $X_{i}^{j}$ into the objective function, get the value $Z_{j}(j=1,2, \cdots, N)$. If there is a $\mathrm{N}$ value $Z_{j}\left(Z_{j}<0\right)$, the probability of the structure failure is:

$$
P_{f}=\frac{N f}{N}
$$

Type : $\mathrm{N}$ is the total number of samples; $N_{f}$ is the number of failures.

Assuming that the relationship between the $\mathrm{Z}$ and the random parameter vector of the spindle structure $X=\left(X_{1}, X_{2}, \cdots, X_{N}\right)$ can be described by the two functions of the cross terms, such as the type (2).

$$
\hat{Z}=\alpha_{0}+\sum_{i=1}^{N} \alpha_{i} X_{i}+\sum_{i=1}^{N} \sum_{j=1}^{N} \alpha_{i j} X_{i} X_{j}
$$

Type : $\alpha_{0} 、 \alpha_{i} 、 \alpha_{i j}(i=1,2, \cdots, N ; i=j, \cdots, N)$-undetermined coefficient, a total of $n+1+n(n+1) / 2$.

The failure of the main shaft of the nuclear power CNC wheel groove milling machine can be considered as the maximum bending deformation of the machine tool, which affects the machining accuracy of the milling machine. Assuming that the maximum bending deformation of the main shaft of the design requirements is $L_{\text {Max }}$, the limit state function is: 


$$
h(X)=\hat{Z}-L_{\text {Max }}=\alpha_{0}+\sum_{i=1}^{N} \alpha_{i} X_{i}+\sum_{i=1}^{N} \sum_{j=1}^{N} \alpha_{i j} X_{i} X_{j}-L_{\text {Max }}
$$

This type represents the two states of the structure: $h(X) \leq 0$ the failure state, $h(X)>0$ the security state. (3) the random parameters are independent of each other, the mean matrix and the matrix of the variance are matrix $\mu=\left(\mu_{1}, \mu_{2}, \cdots, \mu_{N}\right), \quad D=\left(D_{1}, D_{2}, \cdots, D_{N}\right)$ and then the:

$$
\left\{\begin{array}{c}
E\left(X_{i}^{2}\right)=E^{2}\left(X_{i}\right)+D\left(X_{i}\right)=\mu_{i}^{2}+D_{i} \\
E\left(X_{i} X_{j}\right)=E\left(X_{i}\right) E\left(X_{j}\right)=\mu_{i} \mu_{j} \\
D\left(X_{i}^{2}\right)=4 \mu_{i}^{2} D_{i}+2 D_{i} \\
D\left(X_{i} X_{j}\right)=\mu_{i}^{2} D_{j}+\mu_{j}^{2} D_{i}+D_{i} D_{j} \\
E[h(X)]=\mu_{g}\left(\mu_{1}, \mu_{2}, \cdots \mu_{N}, D_{1}, D_{2}, \cdots D_{N}\right) \\
D[h(X)]=D_{g}\left(\mu_{1}, \mu_{2}, \cdots \mu_{N}, D_{1}, D_{2}, \cdots D_{N}\right)
\end{array}\right.
$$

Reliability index is defined as:

$$
\beta=\frac{\mu_{g}}{\sqrt{D_{g}}}
$$

Type : $\beta$ - contact factor; $\mu_{\mathrm{g}}$ - Mean value of state function; $D_{\mathrm{g}}$ - Variance of state function;

It can be obtained from reliability by $h(X)$ the normal distribution.

$$
R=\Phi(\beta)
$$

Type : $\phi(\bullet)$ standard normal distribution function.

Using Monte Carlo simulation theory to calculate the reliability of the principal axis, the sensitivity of the mean matrix $\mu$ and covariance matrix $D$ of the basic random parameter vector is:

$$
\left\{\begin{array}{l}
\frac{\partial R}{\partial \mu^{T}}=\frac{\partial R}{\partial \beta}\left(\frac{\partial \beta}{\partial \mu_{g}} \frac{\partial \mu_{g}}{\partial \mu^{T}}+\frac{\partial \beta}{\partial D_{g}} \frac{\partial D_{g}}{\partial \mu^{T}}\right) \\
\frac{\partial R}{\partial \mathbf{D}^{T}}=\frac{\partial R}{\partial \beta}\left(\frac{\partial \beta}{\partial \mu_{g}} \frac{\partial \mu_{g}}{\partial \mathbf{D}^{T}}+\frac{\partial \beta}{\partial D_{g}} \frac{\partial D_{g}}{\partial \mathbf{D}^{T}}\right)
\end{array}\right.
$$

Type :

$$
\left\{\begin{array}{l}
\frac{\partial R}{\partial \beta}=\psi(\beta) \\
\frac{\partial R}{\partial \mu_{g}}=\frac{1}{\sqrt{D_{g}}} \\
\frac{\partial \beta}{\partial D_{g}}=-\frac{\mu_{g}}{2} D_{g}^{-1.5} \\
\frac{\partial \mu_{g}}{\partial \mu^{T}}=\left(\frac{\partial \mu_{g}}{\partial \mu_{1}}, \frac{\partial \mu_{g}}{\partial \mu_{2}}, \ldots, \frac{\partial \mu_{g}}{\partial \mu_{N_{R}}}\right)^{T} \\
\frac{\partial \mu_{g}}{\partial \mathbf{D}^{T}}=\left(\frac{\partial \mu_{g}}{\partial D_{1}}, \frac{\partial \mu_{g}}{\partial D_{2}}, \ldots, \frac{\partial \mu_{g}}{\partial D_{N_{R}}}\right)^{T} \\
\frac{\partial D_{g}}{\partial \mu^{T}}=\left(\frac{\partial D_{g}}{\partial \mu_{1}}, \frac{\partial D_{g}}{\partial \mu_{2}}, \ldots, \frac{\partial D_{g}}{\partial \mu_{N_{R}}}\right)^{T} \\
\frac{\partial D_{g}}{\partial \mathbf{D}^{T}}=\left(\frac{\partial D_{g}}{\partial D_{1}}, \frac{\partial D_{g}}{\partial D_{2}}, \ldots, \frac{\partial D_{g}}{\partial D_{N_{R}}}\right)^{T}
\end{array}\right.
$$

\section{Reliability analysis of the finite element of the principal axis}

The reliability analysis of the main shaft of the nuclear power plant was carried out by using the finite element design module of PDS, the basic flow chart of the ANSYS is shown in Figure 2. 
Mainly divided into the following three steps: first generate the analysis file, and then enter the reliability analysis phase, again to analyze the results of post processing.

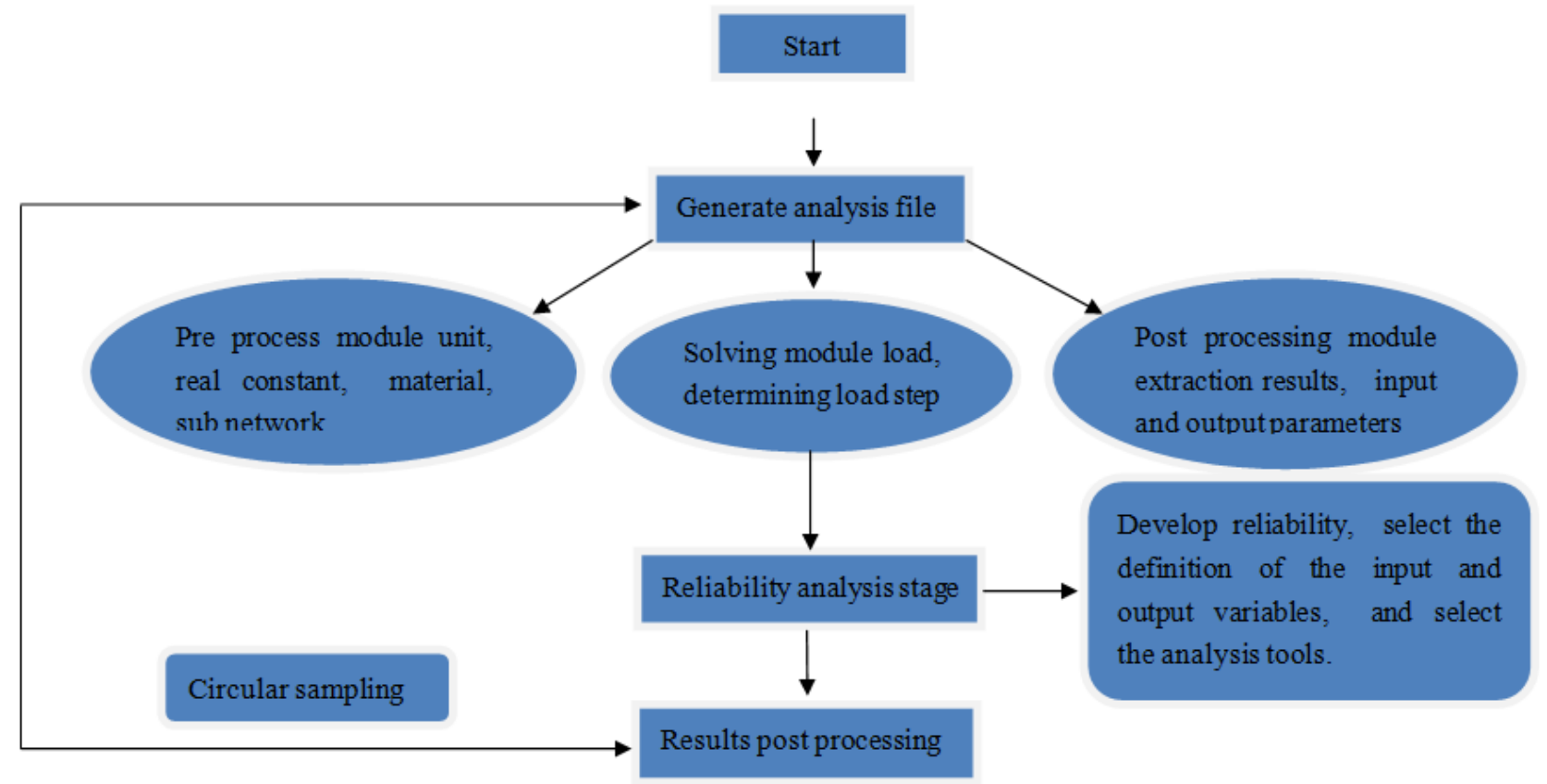

Fig 2 PDS calculation process

(1) Generate analysis file. Analysis file is a critical part of the reliability analysis. The file needs to be written in APDL language, including preprocessing, solving module, result extraction and so on. Structural analysis program through the repeated execution of the analysis to complete the reliability analysis of the cycle. All the parameters that affect the behavior of the structure are specified as the input variables, and the random parameters are shown in Table 3. Parameters corresponding to the analysis of the structural behavior (maximum stress or maximum deformation, etc.) are formulated as the output results.

Tab 3 random parameter list

\begin{tabular}{cccc}
\hline Parameter name & Distribution type & Mean value & Standard deviation \\
\hline $\begin{array}{c}\text { Modulus of elasticity } \\
\text { (EX1) }\end{array}$ & GAUSS & 200GPa & 10GPa \\
\hline Torque (T) & GAUSS & $15000 \mathrm{~N}$ & 300 \\
\hline Diameter (D) & GAUSS & $0.22 \mathrm{~m}$ & $0.008 \mathrm{~m}$ \\
\hline
\end{tabular}

(2) Reliability analysis stage. This phase mainly includes: to enter the reliability analysis file, select the correlation coefficient between the input variables and the input variables, determine the distribution type, the distribution function and the parameters of the input variables, and specify the output variables, the selection of the analysis tools and methods, and the reliability cycle.

(3) Results of post processing. Display, drawing a histogram of the design variables, drawing failure probability distribution function, to determine the correlation coefficient matrix and sensitivity analysis of structure reliability analysis of input and output variables between the sampling process.

Through the above, according to the failure criterion of the spindle rigidity, the maximum deformation of the wheel groove milling machine is not allowed to exceed the allowable deformation, then the limit state function is defined as:

$$
Z(x)=[L]-l_{\max }
$$

If $Z(x)>0$, system security, otherwise failure. Here set $[\mathrm{L}]=0.12 \mathrm{~mm}$, the use of Monte Carlo method, sampling 500 times, after calculation can get the $\mathrm{Z}$ value of the sample. The 
reliability analysis of the main shaft of a nuclear power plant with the finite element method is as follows. The probability distribution function of the 3 parameters is obtained:

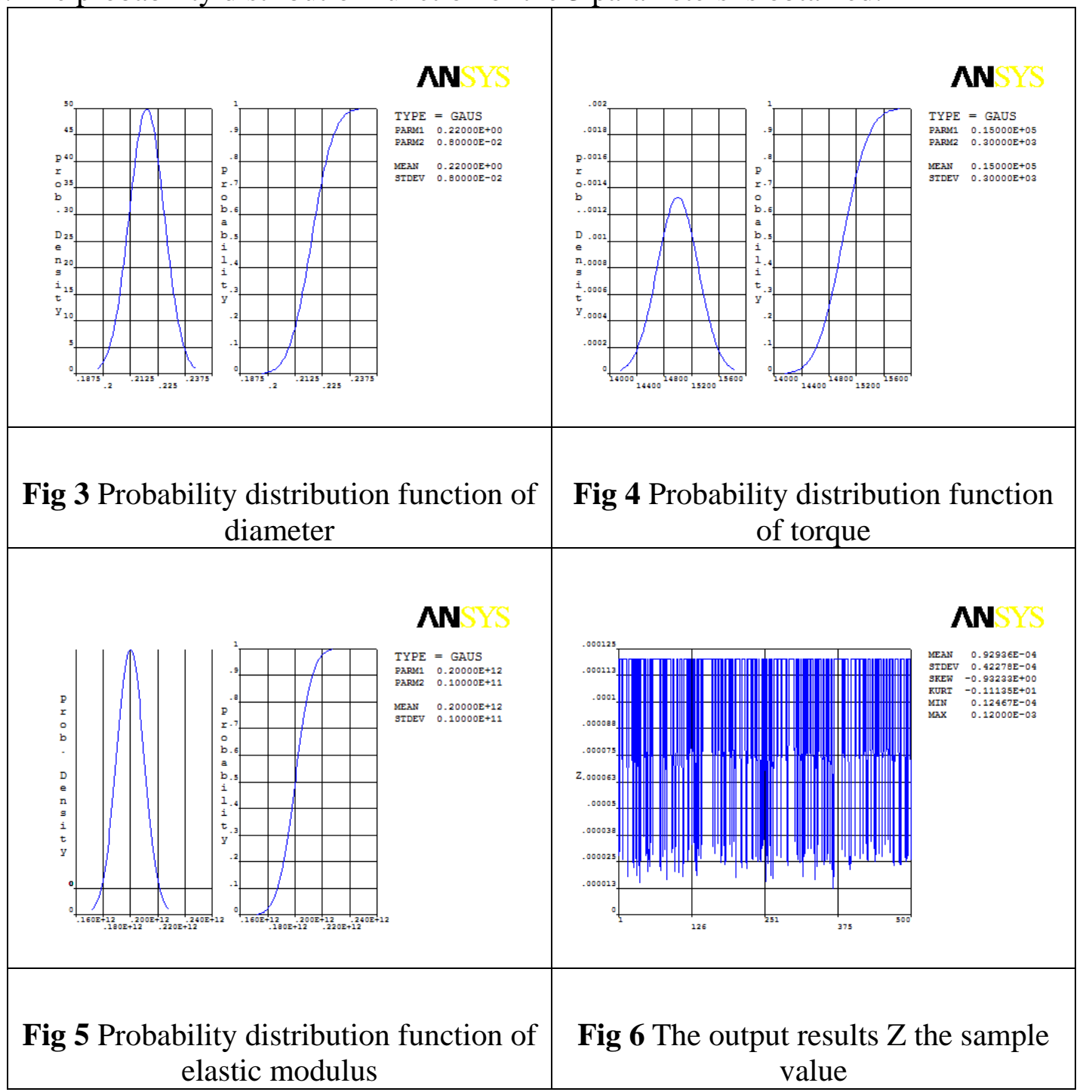

The histogram of the elastic modulus, the diameter and the torque are calculated respectively, which can test the number of cycles. From the histogram can be seen, the curve is smooth, sampling enough.

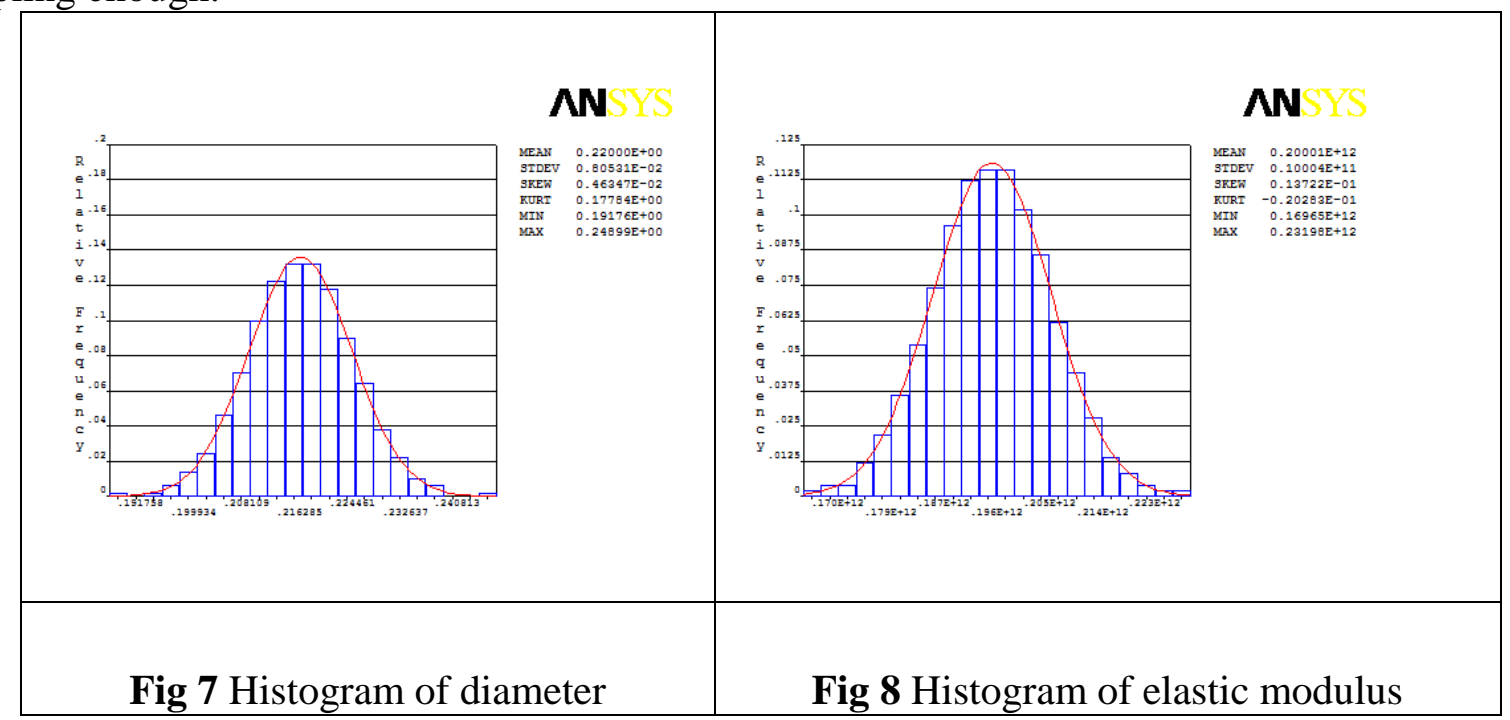




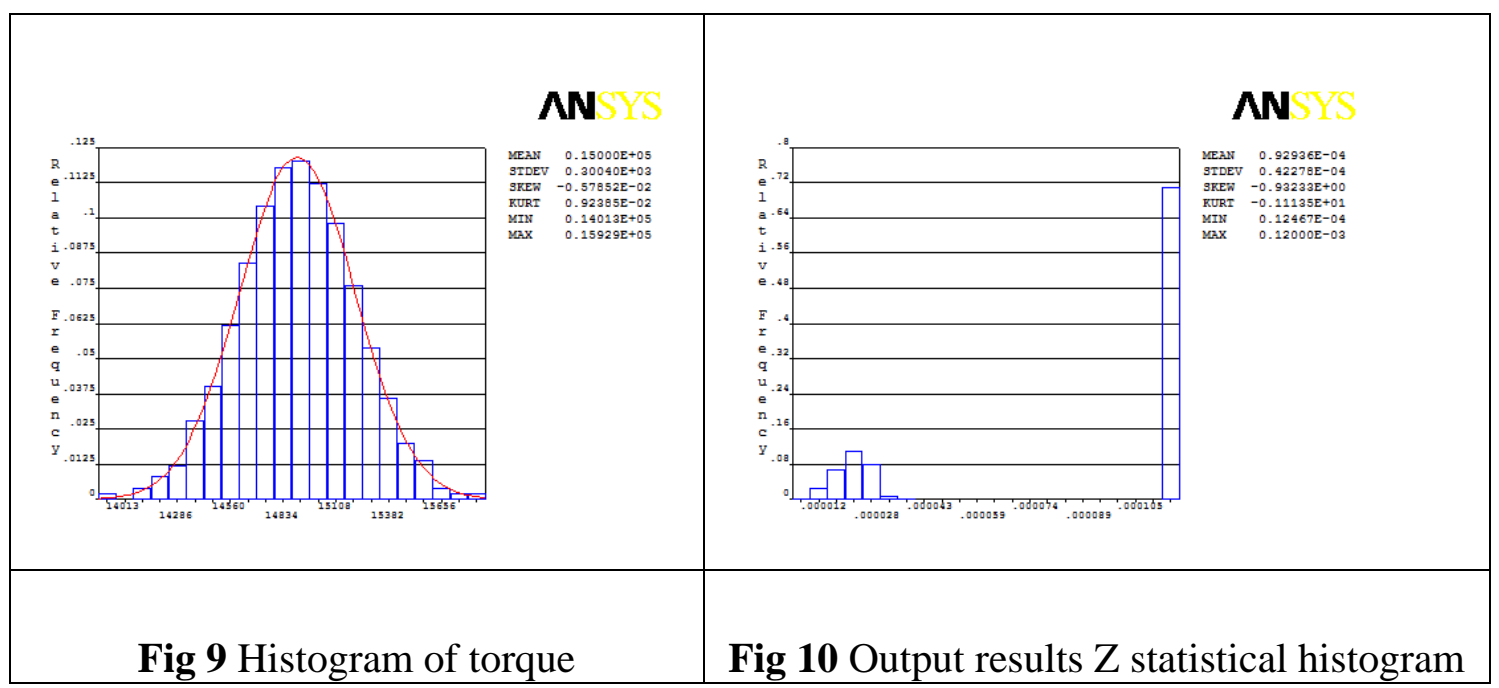

For the Monte Carlo method, the cumulative distribution curves of the three parameter variables and the output results are obtained by setting the confidence probability of $95 \%$.

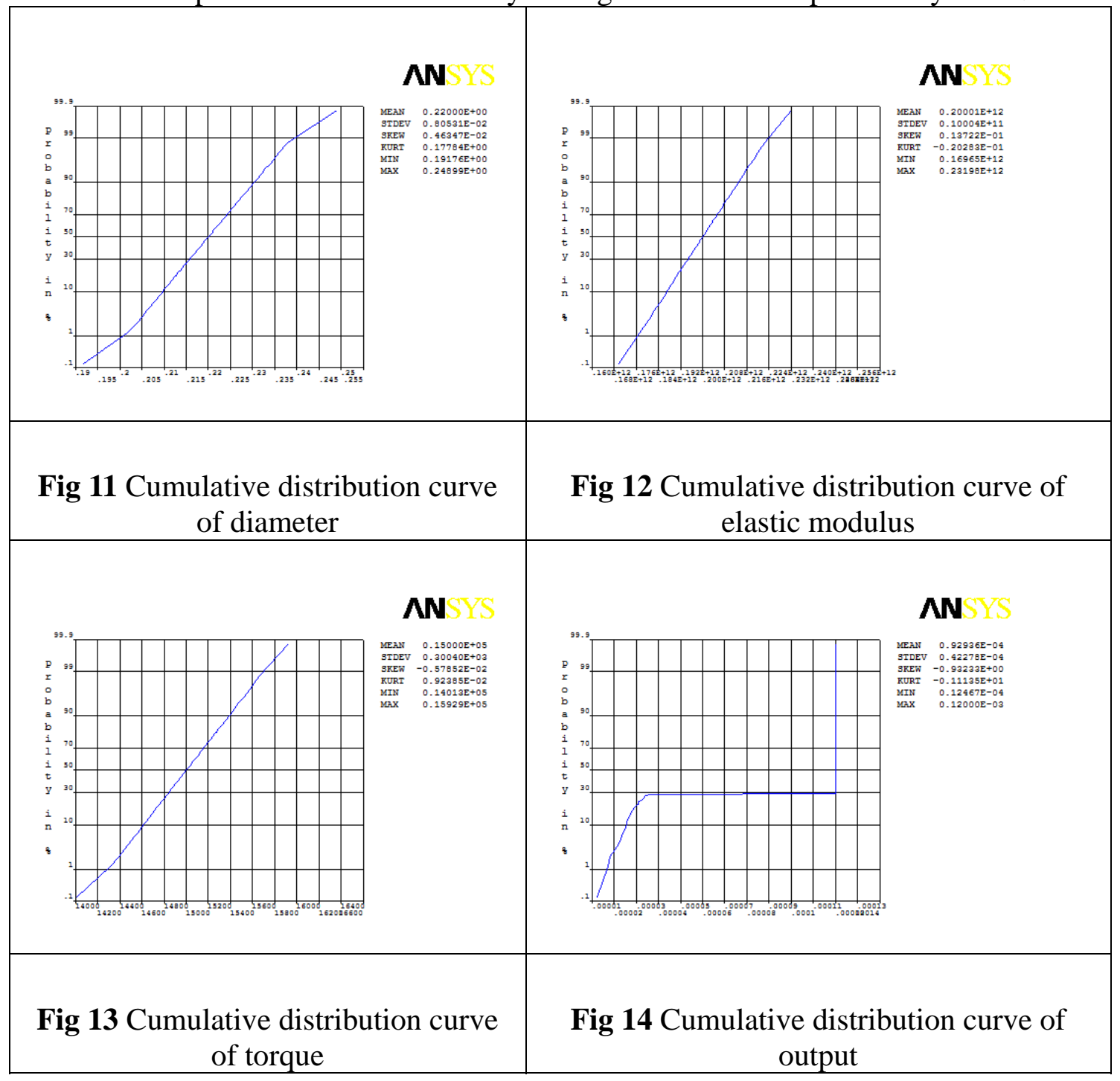

Through the above analysis, we can get the influence of the torque, the diameter, the elastic modulus on the main shaft deformation, so as to obtain their sensitivity. 


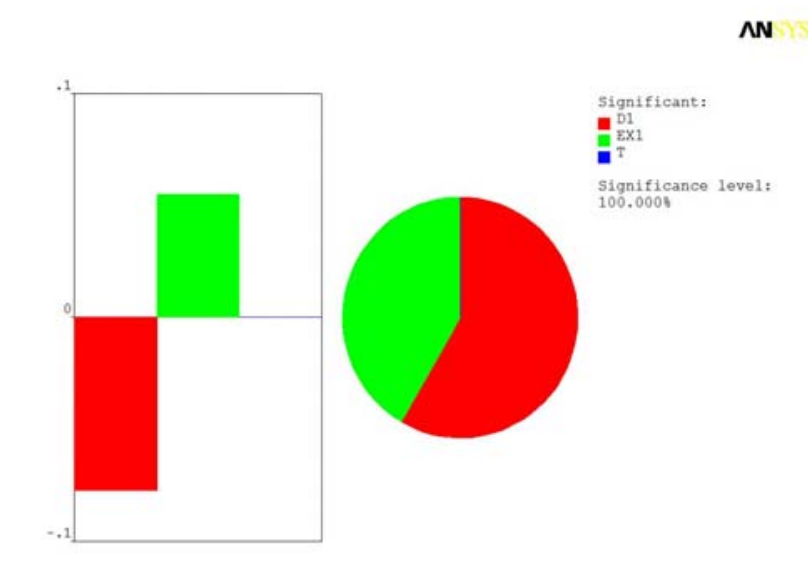

Fig 15 Sensitivity distribution of each parameter

\section{Summary}

From Figure 15, we can see that the main factors affecting the deformation of the main factors are the diameter, the ratio of $62 \%$, the second is the elastic modulus, the last is the torque, the deformation of the spindle is very small relative to the three factors. This is mainly due to the small change of the torque. Finally, in the case of the confidence level is $95 \%$, the sample value of the $\mathrm{Z}$ from the output results can be seen that the reliability of the main shaft is $100 \%$, the value of the sample is greater than zero, that is qualified.

\section{References}

[1] Liu W K.Variational approach to probabilistic finite elements·J·Eng· Mech, ASCE, 1988, 114(12): 2 115 2 131.

[2] Zhang Q, L·Pei U.Random finite element analysis for stochastical responses of structures· Computer \&Structures, 1997, 62(4): 611 616

[3] Coutrears.H, “The Stochastic Finite Element Method”, Computer and Structure, $\quad 1980.12$.

[4] Angela Adamyan, David He .Analysis of sequential failures for assessment of reliability and safety of manufacturing systems [J]. Reliability Engineering and System Safety, 2002, 76(3): 227-236

[5] Theodor Ira Freiheit.Reliabiliy and productivity of reconfigurable manufacturing systems [D].Michigan, USA: University of Michigan 2003

[6] K. Das. A comparative study of exponential distribution vs Weibull distribution in machine reliability analysis in a CMS designs [J]. Computers \& Industrial Engineering, 2008, 54: 12-33

[7] Sun Zhili, Guo Yu, Ji Shi. Research on Mechanical Reliability Growth Model for Complete Failure Data [J]. Advanced Material Research, 2010(118-120):536-540

[8] Zhang Xianpo, Shang Jianzhong, Wang Zhou. Study on simulation reliability test of machining center [J].Advanced Mechanics and Materials, 2010(34-35) : 360-364

[9] Zhang Xianpo, Shang Jianzhong, Wang Zhou. Measures for improving reliability of the machining center [J].Advanced Mechanics and Materials, 2010(34-35) : 572-532 